\title{
High-resolution imaging using ultrasound-modulated optical tomography
}

Sava Sakadzic, Konstantin Maslov, Jun Li, Vikram K. Kinra, Lihong V. Wang

Sava Sakadzic, Konstantin Maslov, Jun Li, Vikram K. Kinra, Lihong V. Wang, "High-resolution imaging using ultrasound-modulated optical tomography," Proc. SPIE 5320, Photons Plus Ultrasound: Imaging and Sensing, (12 July 2004); doi: 10.1117/12.530890

SPIE. Event: Biomedical Optics 2004, 2004, San Jose, CA, United States 


\title{
High resolution imaging using ultrasound-modulated optical tomography
}

\author{
Sava Sakadžić ${ }^{a}$, Konstantin Maslov ${ }^{b}, \operatorname{Jun~Li}^{a}$, Vikram K. Kinra ${ }^{b}$ and Lihong V. Wang ${ }^{a}$ \\ ${ }^{a}$ Optical Imaging Laboratory, Department of Biomedical Engineering, Texas A\&M University, \\ College Station, Texas 77843-3120 \\ ${ }^{b}$ Wave Propagation and Damping Laboratory, Aerospace Engineering Department, \\ Texas A\&M University, College Station, Texas 77843
}

\begin{abstract}
We present an implementation of ultrasound-modulated optical tomography that has the potential to provide high resolution images of tissue structures at a penetration depth of several millimeters. Light and pulsed ultrasound are focused on an approximately $100 \mu \mathrm{m}$ wide area below the sample surface. With this configuration, the length of the ultrasonic pulses determines the axial resolution, and the lateral resolution results from the width of the ultrasonic beam at the focus. Diffuse light reflected from the sample is collected into a fiber and the modulated component is separated from the background by a confocal Fabry-Perot interferometer. Using this setup, high contrast images are obtained of $100 \mu \mathrm{m}$ wide pieces of hair that are buried one millimeter below the surface of the tissue-mimicking sample. It is the first time, to the authors' knowledge, that images with such high resolution have been obtained using ultrasound-modulated optical tomography in the reflection mode.
\end{abstract}

Keywords: ultrasonic modulation, acousto-optical tomography, high resolution, multiply scattered light

\section{INTRODUCTION}

The optical properties of tissues in the visible and near infrared parts of the spectrum are related to the molecular structures of the tissues. Therefore, they offer great potential for the detection of tissue functions and abnormalities. In addition, radiation at these wavelengths is nonionizing and more appropriate for medical applications. Great effort has been made by many groups of researchers in the recent past to develop new imaging modalities based on the optical properties of biological tissues in the visible and near infrared regions.

The resolution in the pure optical imaging of thick tissue samples is limited due to the high levels of scattering and absorption of light. Acousto-optical tomography ${ }^{1}$ (AOT) is a new hybrid technique, proposed to provide better resolution in the optical imaging of thick biological samples. This technique combines ultrasonic resolution and optical contrast that is based on the differences in optical properties among different types of tissues. In general, the collective motion of the scatterers and the periodical changes in the index of refraction that are generated by focused ultrasound produce fluctuations in the intensity of the speckles formed by the multiply scattered light. ${ }^{2-7}$ As a result, the alternating current of a photo detector at the frequency of ultrasound carries information about the optical properties of tissue from the region of the intersection of the optical and acoustical waves. In AOT, the maximum resolution and the maximum imaging depth are scalable with an ultrasound frequency.

In practice, the detection of AOT signals is nontrivial. Light emerging from a tissue sample creates a strong speckle pattern with very little correlation between the phases of the individual speckles. At the same time, due to light diffusion, the mismatch between the emission and detection etendue is very large in practical applications. Several different schemes of detection ${ }^{3,4,8-25}$ for AOT have been proposed in the last few years. One especially promising detection technique is a parallel speckle detection scheme using a CCD camera, ${ }^{10-12,16,17,21-23,25}$ since detection over many CCD pixels provides a larger etendue and a higher signal-to-noise ratio than the simple heterodyning method which uses only one square-low detector.

For many reasons, an optical imaging technique that is capable of providing resolution better than 100 $\mu m$ with an imaging depth of several millimeters is highly desirable. In this work, for the first time to our knowledge, we explore the possibility of using AOT for high-resolution tissue imaging. Additionally, we propose 


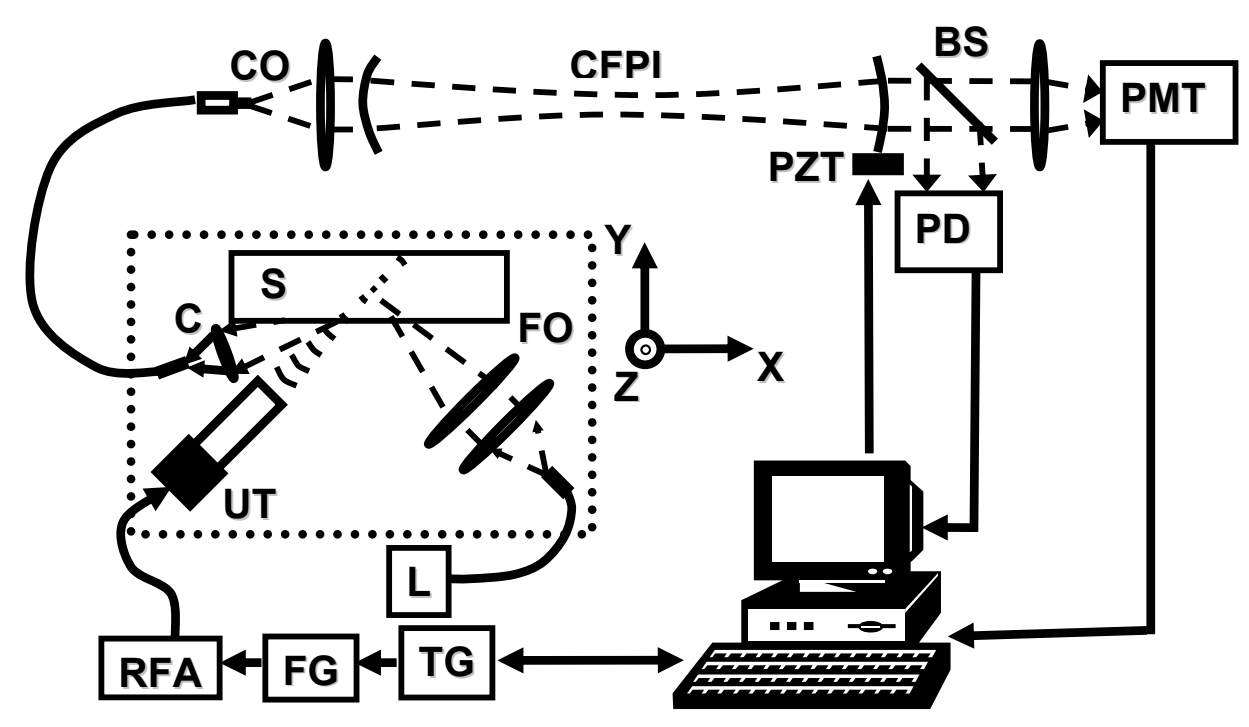

Figure 1. Arrangement for high-resolution AOT imaging: L, laser; TG, trigger generator; FG, function generator; RFA, radio-frequency amplifier; UT, ultrasonic transducer; FO, focusing optics; C, collecting optics; S, sample; CO, coupling optics; CFPI, confocal Fabry-Perot interferometer; BS, beam splitter; PMT, photomultiplier tube; PD, photo detector.

another scheme for the detection of AOT signals using a large confocal Fabry-Perot interferometer (CFPI) as the filter. This detection technique has already been successfully implemented in laser ultrasonics where very similar detection problems are encountered. ${ }^{26}$ A large CFPI has high resolution, the capability to process many speckles, and a large etendue. Moreover, the small time constant of the cavity allows for the real time tracking of ultrasound pulses using the acousto-optical effect, which is not possible with current CCD cameras.

Our preliminary results show that images of $100 \mu \mathrm{m}$ wide pieces of dark hair placed one millimeter below the surface of tissue-mimicking samples are obtainable with very high contrast.

\section{EXPERIMENTAL SETUP}

Our experimental setup, shown in Fig. 1, was organized as follows: the ultrasound beam and the light beam were focused at the same spot below the sample surface, with a $90^{\circ}$ angle between the ultrasound propagation direction and the light beam axis. The plane that was defined by the ultrasound axis and the light axis was perpendicular to the sample surface. A $25 \mathrm{MHz}$ focusing transducer (UT) was used to generate the ultrasound signal (Panametrics V324, lens diameter $D=6.25 \mathrm{~mm}$, focal length $F=12.5 \mathrm{~mm}$ ). The diameter of the ultrasonic beam at the focal point was around $150 \mu \mathrm{m}$. In the optical part of the setup, the light from the diode pumped Nd:YAG laser (L) with a very long coherence length (Adlas DPY 325, $532 \mathrm{~nm}$ - wavelength, coherence length $>50 \mathrm{~m}$ ) was first coupled into a multimode fiber, and then focused onto an approximately 100 $\mu m$ wide spot below the sample surface. The average power delivered to the sample was $15 \mathrm{~mW}$. Sample (S) was mounted on a three axis $(X, Y$, and $Z)$ manual translational stage. To better couple the ultrasound beam with the sample, the sample was immersed in water. We also immersed the light focusing optics (FO) and the light collecting optics (C) in the same water tank.

Diffusely reflected light from the sample was collected by the light collecting optics and coupled into the multimode fiber (400 $\mathrm{mm}$ core diameter). The light was further coupled into a scanning confocal Fabry-Perot interferometer that was operated in transmission mode (Lisor RT, Ultraoptec, cavity length $50 \mathrm{~cm}$, free spectral range $150 \mathrm{MHz}$, finesse $>20$, etendue $0.1 \mathrm{~mm}^{2} s r$ ). The signal sampled by the beam splitter (BS) provided information for controlling the position of the one CFPI mirror. Our software, which was written in LabVIEW, controlled the precise movement of the interferometer mirror so that the length of the cavity was always tuned to the frequency of the ultrasound-modulated light $(25 \mathrm{M} \mathrm{Hz}$ away from the laser light frequency). The same software also managed the data processing and all of the other sequences of the instrument control signals. A 


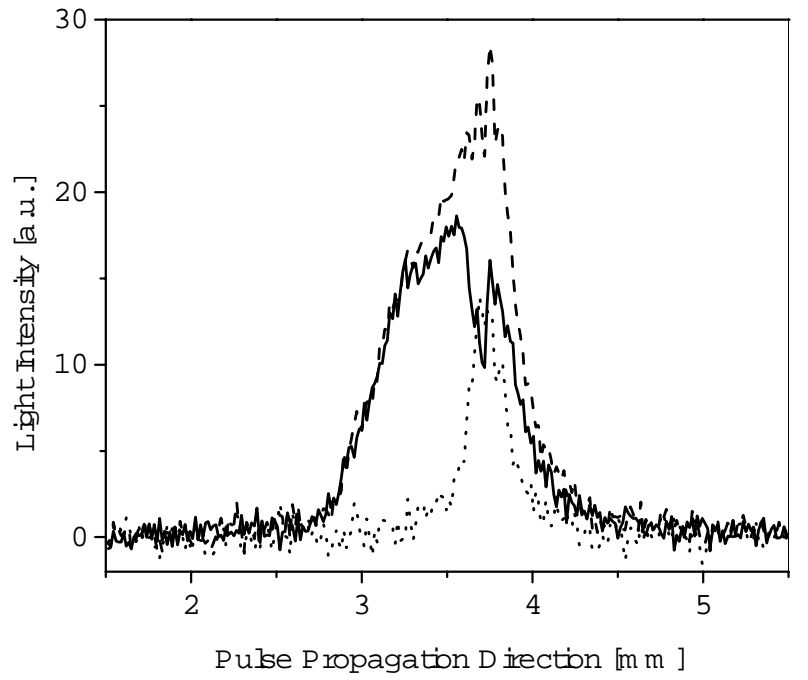

Figure 2. Ultrasound-modulated light intensity measured during the ultrasound pulse propagation through the sample. The time intervals along the ultrasound pulse propagation direction are converted into positions according to the speed of sound in tissue. Dashed line - modulated intensity when hair was away from the ultrasonic axis. Solid line - modulated intensity when hair was exactly at the ultrasonic focus. Dotted line - difference between previous two signals.

photomultiplier tube (Hamamatsu R928) positioned behind the CFPI acquired the light filtered by the interferometer, and the signal was recorded with a fast data acquisition board (GAGE, CompuScope 14100).

The signal was acquired in the following way: the trigger generator (TG) triggered the ultrasound pulse generation and, with an appropriate delay, the acquisition of the signal from the PMT tube. One sinus wave at a frequency of $25 \mathrm{MHz}$ was produced by a function generator (FG) and amplified by a RF amplifier (RFA) to $150 \mathrm{Vpp}$. Due to the limited bandwidth of the transducer, the ultrasound pulse contained several cycles with a total length around $150 \mu \mathrm{m}$. Since the resonant frequency of the FP cavity coincided with the frequency of the ultrasound modulated light, the signal acquired by the PMT tube during the time of the ultrasound propagation through the sample represented the ultrasound modulated intensity variations along the ultrasound pulse propagation. Our long memory fast data acquisition board allowed us to store the data from 1000 ultrasound pulses before requiring transfer to computer memory.

\section{RESULTS AND DISCUSSION}

In the first experiment (Fig. 2), we prepared the sample using chicken breast tissue. A $100 \mu m$ wide dark hair was placed $1 \mathrm{~mm}$ below the surface of the sample, and then the common focal spot of the ultrasound and the light beams was positioned at the same depth $(1 \mathrm{~mm})$ below the sample surface. In the first measurement, the sample was positioned so that the piece of hair was away from the ultrasonic axis. The measurement (dashed line on Fig. 2) shows that the light radiance within the sample and the profile of the ultrasound beam intensity determined the shape of the modulated intensity signal, with the maximum corresponding to the position of the light and the ultrasound focus. In the second measurement, the sample was positioned so that the piece of hair was exactly at the ultrasound focus. From the measurement data (solid line on Fig. 2), we see that differences in the optical properties of the hair and the surrounding chicken breast tissue created a large decay in the modulated light intensity during the time interval when the ultrasound pulse passed through the hair. Full recovery of the modulated light intensity after the ultrasound pulse exited the hair presented evidence that contrast in the measurement was based primarily on the optical properties of the sample and the object, and not on the influence of the sample on the ultrasound pulse.

In the second experiment, a gelatine sample was prepared using intralipid solution to mimic the tissue scattering properties. Several dark pieces of hair of the same $100 \mu \mathrm{m}$ diameter were placed $1 \mathrm{~mm}$ below the 

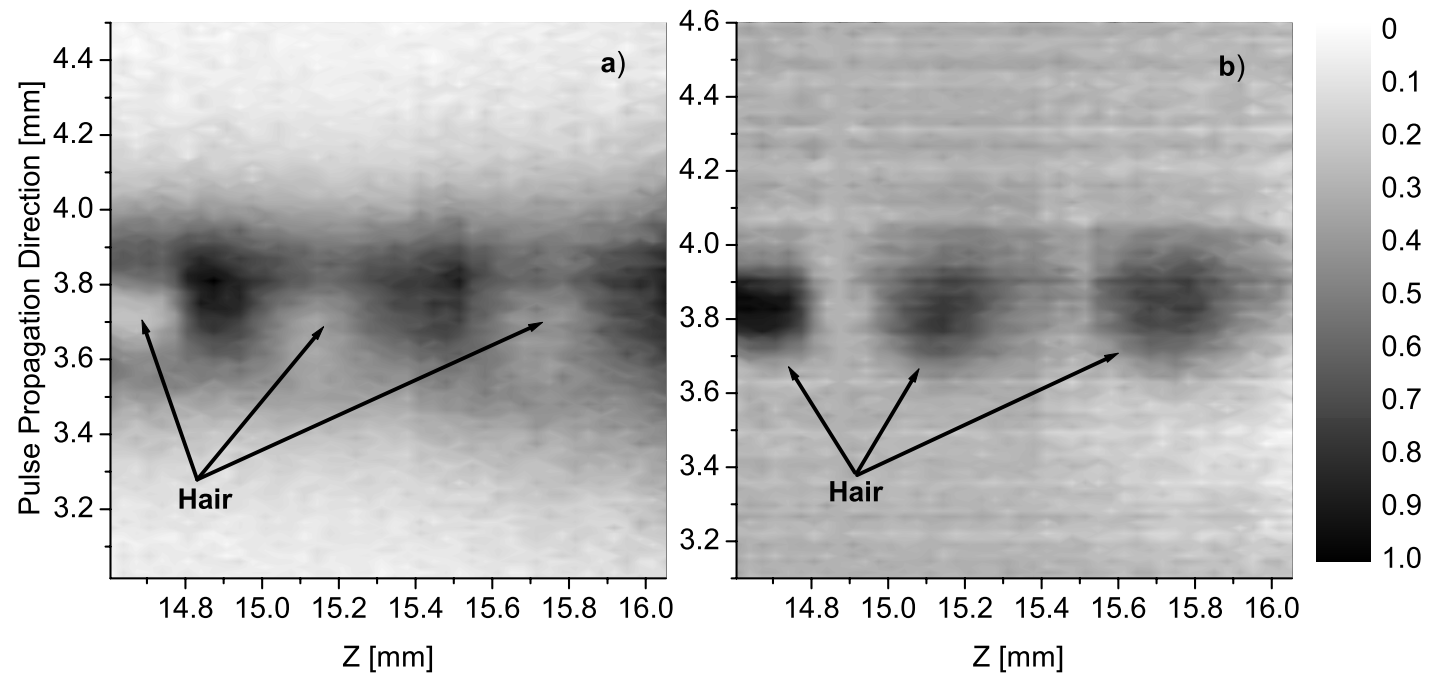

Figure 3. 2D ultrasound-modulated intensity map of a tissue mimicking sample with several $100 \mu m$ wide dark hair pieces placed $1 \mathrm{~mm}$ below the sample surface. Beside the long piece of hair on the very left on the figures, the other two pieces are $150 \mu \mathrm{m}$ long and placed along the $Z$ axis in continuity with the long piece of hair. a) signal obtained directly from the PMT. b) intensity map from the case a) subtracted from the signal obtained along the pulse propagation direction when the ultrasound axis was away from any hair piece.

sample surface. The system of hair samples consists of one long hair piece placed along the $Z$ direction, and in continuation with it, several $150 \mu \mathrm{m}$ pieces separated a few hundred micrometers apart. The long hair piece was chosen in order to speed up the time necessary to locate the position of the short hair pieces. Once the common focal spot of the ultrasound and light was positioned at the center of the long hair piece, the sample was scanned along the $Z$ direction. For each $Z$ position, the signal along the ultrasound pulse propagation direction was obtained in the same way as in the measurement presented in Fig. 2. After collecting data for many positions along the $Z$ axis, the two dimensional ultrasound modulated intensity map presented in Fig. 3 was created. Fig. 3a presents the signal directly obtained from the PMT tube. The pieces of hair appear in Fig. 3a as decay in the modulated intensity, similar to the signal behavior in Fig. 2. Fig. 3b presents the same data, with the difference that all of the values are subtracted from the signal profile at the position where the ultrasound axis was away from any hair piece. The positions of the pieces of hair are marked on the image. The gaps between them are clearly visible with very high contrast.

\section{CONCLUSION}

Using a large confocal Fabry-Perot interferometer to isolate ultrasonically modulated light from background un-modulated light at high ultrasound frequencies allowed us to significantly improve the SNR in acoustically resolved laser imaging. At the same time, the fast time response of the instrument allowed for tracking of the ultrasonic pulses in real time and provided an opportunity to obtain resolution along the ultrasound axis directly without implementing a frequency sweep technique or computer tomography reconstruction. We presented a 1D image of a $100 \mu \mathrm{m}$ wide piece of dark hair placed $1 \mathrm{~mm}$ below the surface of the chicken breast tissue sample, and a 2D image of similar hair placed $1 \mathrm{~mm}$ below the surface of the tissue mimicking sample. The pieces of hair in both cases were clearly visible with very high contrast. Our preliminary results show that high-resolution imaging is one promising direction for AOT. Further investigation is necessary to explore the maximum capabilities of and the application directions for this technique. 


\section{REFERENCES}

1. F. A. Marks, H. W. Tomlinson, and G. W. Brooksby, "A comprehensive approach to brest cancer detection using light: photon localization by ultrasound modulation and tissue characterization by spectral discrimination," in Proc. Soc. Photo-Opt. Instrum. Eng., 1888, pp. 500-510, 1993.

2. W. Leutz and G. Maret, "Ultrasonic modulation of multiply scattered-light," Physica B 204, pp. 14-19, 1995.

3. M. Kempe, M. Larionov, D. Zaslavsky, and A. Z. Genack, "Acousto-optic tomography with multiply scattered light," J. Opt. Soc. Am. A 14, pp. 1151-1158, 1997.

4. G. D. Mahan, W. E. Engler, J. J. Tiemann, and E. G. Uzgiris, "Ultrasonic tagging of light: Theory," in Proc. Natl. Acad. Sci. USA, 95, p. 14015, 1998.

5. L.-H. V. Wang, "Mechanisms of ultrasonic modulation of multiply scattered coherent light: an analytic model," Phys. Rev. Lett. 87, pp. 043903-(1-4), 2001.

6. L.-H. V. Wang, "Mechanisms of ultrasonic modulation of multiply scattered coherent light: a monte carlo model," Opt. Lett. 26, pp. 1191-1193, 2001.

7. S. Sakadzic and L.-H. V. Wang, "Ultrasonic modulation of multiply scattered coherent light: an analytical model for anisotropically scattering media," Phys. Rev. E 66, pp. 026603-(19), 2002.

8. L.-H. V. Wang, S. L. Jacques, and X. Zhao, "Continuous-wave ultrasonic modulation of scattered laser light to image objects in turbid media," Opt. Lett. 20, pp. 629-631, 1995.

9. L.-H. V. Wang and G. Ku, "Frequency-swept ultrasound-modulated optical tomography of scattering media," Opt. Lett. 23, pp. 975-977, 1998.

10. S. Leveque, A. C. Boccara, M. Lebec, and H. Saint-Jalmes, "Ultrasonic tagging of photon paths in scattering media: parallel speckle modulation processing," Opt. Lett. 24, pp. 181-183, 1999.

11. G. Yao and L.-H. V. Wang, "Theoretical and experimental studies of ultrasound-modualted optical tomography in biological tissue," Appl. Opt. 39, pp. 659-664, 2000.

12. G. Yao, S.-L. Jiao, and L.-H. V. Wang, "Frequency-swept ultrasound-modulated optical tomography in biological tissue by use of parallel detection," Opt. Lett. 25, pp. 734-736, 2000.

13. A. Lev, Z. Kotler, and B. G. Sfez, "Ultrasound tagged light imaging in turbid media in a reflectance geometry," Opt. Lett. 25, pp. 378-380, 2000.

14. E. Granot, A. Lev, Z. Kotler, B. G. Sfez, and H. Taitelbaum, "Detection of inhomogeneities with ultrasound tagging of light," J. Opt. Soc. Am. A-Opt. Image Sci. Vis. 18, pp. 1962-1967, 2001.

15. M. Hisaka, T. Sugiura, and S. Kawata, "Optical cross-sectional imaging with pulse ultrasound wave assistance," J. Opt. Soc. Am. A-Opt. Image Sci. Vis. 18, pp. 1531-1534, 2001.

16. S. Leveque-Fort, "Three-dimensional acousto-optic imaging in biological tissues with parallel signal processing," Appl. Optics 40, pp. 1029-1036, 2001.

17. J. Li, G. Ku, and L.-H. V. Wang, "Ultrasound-modulated optical tomography of biological tissue by use of contrast of laser speckles," Appl. Optics 41, pp. 6030-6035, 2002.

18. H. Li and L.-H. V. Wang, "Autocorrelation of scattered laser light for ultrasound-modulated optical tomography in dense turbid media," Appl. Optics 41, pp. 4739-4742, 2002.

19. J. Selb, L. Pottier, and A. C. Boccara, "Nonlinear effects in acousto-optic imaging," Opt. Lett. 27, pp. 918920, 2002.

20. A. P. Solov'ev, Y. P. Sinichkin, and O. V. Zyuryukina, "Acoustooptic visualization of scattering media," Opt. Spectrosc. 92, pp. 214-220, 2002.

21. J. Li and L.-H. V. Wang, "Methods for parallel-detection-based ultrasound-modulated optical tomography," Appl. Optics 41, pp. 2079-2084, 2002.

22. A. Lev and B. G. Sfez, "Direct, noninvasive detection of photon density in turbid media," Opt. Lett. 27, pp. 473-475, 2002.

23. M. Gross, P. Goy, and M. Al-Koussa, "Shot-noise detection of ultrasound-tagged photons in ultrasoundmodulated optical imaging," Opt. Lett. 28, pp. 2482-2484, 2003.

24. A. Lev and B. G. Sfez, "Pulsed ultrasound-modulated light tomography," Opt. Lett. 28, pp. 1549-1551, 2003. 
25. J. Li, S. Sakadzic, G. Ku, and L.-H. V. Wang, "Transmission and side detection configurations in ultrasoundmodulated optical tomography of thick biological tissues," Appl. Optics 42, pp. 4088-4094, 2003.

26. J.-P. Monchalin, "Optical detection of ultrasound at a distance using a confocal fabry-perot interferometer," Appl. Phys. Lett 47, pp. 14-16, 1985. 